Research Article

\title{
Modeling and Parameter Identification of the MR Damper Based on LS-SVM
}

\author{
Cheng Qian (D), Xiaoliang Yin, and Qing Ouyang \\ Jiaxing University, Mechanical and Electrical Engineering College, Zhejiang Jiaxing 314001, China \\ Correspondence should be addressed to Cheng Qian; qc117@sina.com
}

Received 11 November 2020; Revised 27 January 2021; Accepted 30 January 2021; Published 17 February 2021

Academic Editor: Yue Wang

Copyright (C) 2021 Cheng Qian et al. This is an open access article distributed under the Creative Commons Attribution License, which permits unrestricted use, distribution, and reproduction in any medium, provided the original work is properly cited.

\begin{abstract}
In order to identify the nonlinear characteristics of the magnetorheological (MR) damper applied in multi-DOF vibration reduction platforms in the aerospace field in the modeling process, the least square support vector machine (LS-SVM) method is adopted, because LS-SVM can handle small-sample, high-dimensional characteristic problems. Firstly, the theory of the modeling method based on LS-SVM was illustrated including the genetic algorithm (GA) optimization method. Secondly, the characteristic curve of the MR damper was tested based on different conditions. Then, the current and historical input displacement, velocity, and current and the historical output are taken as the input of the LS-SVM model and the damping force of the current output is taken as the output of the model for model training. Meanwhile, the genetic algorithm is introduced to optimize the parameters of the LS-SVM model which affect the accuracy of the model, the penalty factor $c=16.48$, and the kernel parameter $\sigma=3.39$ after optimization. Finally, in order to verify the method adopted in the paper, the Simulink model was simulated in certain input conditions; by comparing the simulation and experimental values of this model, it is found that the maximum error is within $10 \mathrm{~N}$ and the average error is around $0.89 \mathrm{~N}$, which is similar to the accuracy obtained in other works of literature, and the correctness of this model is verified.
\end{abstract}

\section{Introduction}

The magnetorheological damper is mainly made of magnetorheological fluid, and its rheological properties drastically change in millisecond time according to different excitation magnetic fields. Its advantages are shown as follows: simple structure, rapid response, continuous adjustable damping force, and good controllability. So it is a kind of excellent intelligent semiactive damping device, which is widely used in bridges, cars, buildings, aerospace, and other areas of the vibration reduction field [1-3]. However, the magnetorheological fluid (MRF) property will have multiple field coupling affected by the fluid itself, and the excitation magnetic field in the case of the rheological properties drastically change. Therefore, when analyzing the damping force characteristics of the magnetorheological damper, it can be found that its dynamic characteristics have obvious nonlinear and hysteresis characteristics, and its mechanical model has complex nonlinearity as a result [4]. Due to the existence of these inherent nonlinear hysteresis characteristics, the repeatability and control accuracy will be reduced in the control process of the MR damper. And the real-time transient response and control results cannot meet the set goals, so it is difficult to promote the application of the MR damper in the highprecision space multidimensional vibration reduction field. Therefore, the accurate mechanical model is the basis for improving the control accuracy and transient response accuracy of the MR damper, establishing effective control methods, and giving full play to the semiactive performance of the MR damper.

At present, the dynamic model of the MR damper has been studied extensively and deeply by scholars at home and abroad. These models can be divided into two categories. The first category is called the parametric model, whose parameters have some physical meanings, mainly including the classic Bingham model [5], Dahl model [6], Bouc-Wen 
model $[7,8]$, nonlinear double-viscous model [9], and sigmoid model $[10,11]$. The second category is called the nonparametric model, whose model parameters have no physical meaning, mainly including the improved LuGre friction model [12], RC model [13], binary-coded discrete CSO and ELM [14], and mixed intelligent model [15, 16]. Among them, although the Bingham model can well express the force-displacement characteristics of the magnetorheological damper, it cannot accurately express the force-velocity characteristics, especially in the low-speed region. The Bouc-Wen model has been widely used, but a series of parameters need to be measured in the process of application. In general, in order to improve the accuracy, it will be combined with other intelligent algorithms for parameter identification. As the double-viscous model is a piecewise function, it cannot accurately fit the force-velocity characteristics. Generally speaking, some idealized operations will be carried out in the design and research process of the parametric model, and the calculated parameters may be contrary to the reality, which will also have a certain impact on the results. However, the study of the nonparametric model mainly relies on the input and output data of the MR damper under different conditions in the test process for analysis and training. For example, Zamani et al. proposed an MSDP model, which obtained force-displacement and force-velocity data from different excitation amplitude, frequency, and current values for parameter identification of the model and achieved high accuracy [17]. Although the nonparametric model has high precision, it has a high requirement on the sample data volume, and its training processes, such as fuzzy logic determination, neural network, and fuzzy neural network processes, are quite complicated.

Due to the different excitation amplitude and frequency values and the different magnitude values of current, the output characteristics of the magnetorheological damper will change. In the actual nonparametric modeling process, not all cases can be included, so the experimental data available for modeling is limited; at the same time, the output characteristics of the magnetorheological damper have strong nonlinear characteristics. The nonparametric modeling process of the MR damper can be summarized as having the characteristics of less training sample data and nonlinear dynamics. In many machine learning languages, SVM (support vector machine) is suitable for small-sample, nonlinear, highdimensional problems, and it has excellent learning ability, which is based on the statistical learning theory and structural risk minimization principle. Based on the limited sample information, it seeks the best compromise between the complexity of the model and the learning ability in order to obtain the best generalization ability [18].

Therefore, this paper takes the magnetorheological dampers applied in multi-DOF vibration reduction platforms in the aerospace field as the research object, analyzes its dynamic nonlinear characteristics, and establishes an accurate simulation model as the target. The main content is as follows. The input and output sample data under the influence of different excitation displacements, velocities, and currents were taken into account for the support vector machine model training of the MR damper. During the train-

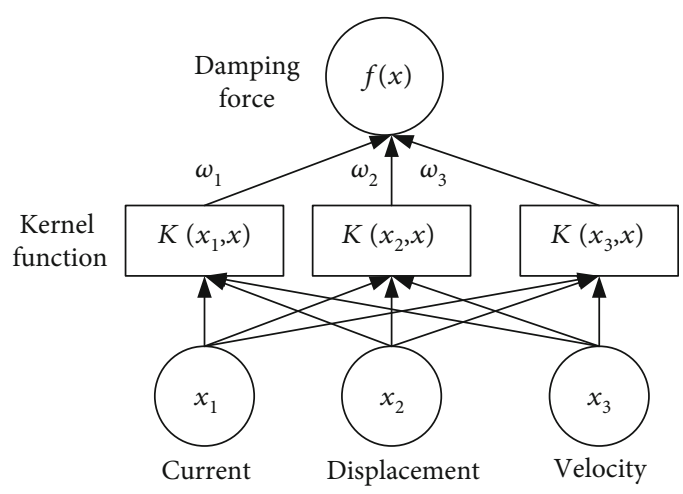

Figure 1: Principle of the SVM model.

ing process, the model parameters of the support vector machine are optimized in order to obtain the accurate output of the model which can be calculated according to different input displacement, velocity, and current intensity values. Finally, the correctness of the MR damper support vector machine model is verified by comparative analysis between the simulation and experimental results. The innovation point of this paper is the LS-SVM model of the MR damper, and the parameters of the LS-SVM model were optimized by the genetic algorithm.

\section{Support Vector Machine Model Theory for the MR Damper}

The core idea of using the support vector machine to build the MR damper model can be summarized as follows. The nonlinear relations between the input displacement, velocity, and current intensity and the output damping force, which affect the characteristics of the magnetorheological damper, are mapped to a high-dimensional feature space by the kernel function of the support vector machine. In this highdimensional feature space, the nonlinear problem is transformed into a linear problem, and the regression function is constructed to find the nonlinear relationship between the input and the output of the training sample. The principle is shown in Figure 1.

In order to build the above SVM model, it can be seen from the input and output characteristics of the magnetorheological damper that its damping force is related to the current and historical current intensity, displacement, and velocity, as well as the historical output. Therefore, the model can be defined as shown in the following equation:

$$
\begin{aligned}
y\left(x_{i}\right) & =\omega^{T} \cdot \varphi\left(x_{i}\right)+b, \\
x_{i} & =\left[d_{1}, d_{2}, \cdots, d_{i}, v_{1}, v_{2}, \cdots, v_{i-1}, I\right],
\end{aligned}
$$

where $\varphi\left(x_{i}\right)$ represents the Hilbert high-dimensional mapping function; $\omega$ represents the weight vector of the hyperplane; $b$ represents the offset value; $x_{i}$ represents the input value of the sample, and it includes the displacement $\left[d_{1}, d_{2}, \cdots, d_{i}\right]$, velocity $\left[v_{1}, v_{2}, \cdots, v_{i-1}\right]$, and magnitude of current $I$; and $y\left(x_{i}\right)$ represents the output value under the current input parameters of the sample set. 
The values of the weight vector $\omega$ and the offset value $b$ are calculated based on the principle of structural risk minimization. Meanwhile, the SVM optimization problem was transformed into a quadratic programming problem; thus, the LS-SVM optimization problem was obtained as shown in the following equation:

$$
\min \frac{1}{2} \omega^{T} \cdot \omega+\frac{c}{2} \sum_{i=1}^{n} \varepsilon_{i}^{2}, \quad \text { s.t. }\left|y_{i}-\omega^{T} \varphi\left(x_{i}\right)-b\right| \leq \varepsilon_{i},
$$

where $c$ represents the penalty factor, $\varepsilon_{i}$ represents the relaxation variable, and $n$ represents the number of sample data.

In order to solve the optimization problem under the inequality constraints, the inequality constraints can be changed into equality constraints and the Lagrangian operator $a_{i}(i=1,2, \cdots, n)$ was used to construct the Lagrangian equation:

$L\left(\omega, \varepsilon_{i}, b, a\right)=\frac{1}{2} \omega^{T} \omega+\frac{c}{2} \sum_{i=1}^{n} \varepsilon_{i}^{2}-\sum_{i=1}^{n} a_{i}\left(\omega^{T} \cdot \varphi\left(x_{i}\right)+b+\varepsilon_{i}-y_{i}\right)$.

To solve the extreme value problem in equation (2), make the partial derivatives of variables $\omega, \varepsilon_{i}, b$, and $a$ in Lagrangian equation (3) equal to 0 ; namely, solve the equations shown in the following equation:

$$
\left\{\begin{array}{l}
\frac{\partial L}{\partial \omega}=\omega-\sum_{i=1}^{n} a_{i} \cdot \varphi\left(x_{i}\right)=0, \\
\frac{\partial L}{\partial \varepsilon_{i}}=c \cdot \varepsilon_{i}-a_{i}=0, \\
\frac{\partial L}{\partial b}=\sum_{i=1}^{n} a_{i}=0, \\
\frac{\partial L}{\partial a_{i}}=\omega \cdot \varphi\left(x_{i}\right)+b+\varepsilon_{i}-y_{i}=0 .
\end{array}\right.
$$

By solving the equations of equation (4) and eliminating the parameters of $\omega$ and $\varepsilon_{i}$, the optimization problem can be transformed into solving a system of linear equations, as shown in the following equation:

$$
\left[\begin{array}{cccc}
0 & y_{1} & \cdots & y_{n} \\
y_{1} & y_{1} y_{1} \varphi\left(x_{1}\right) \varphi\left(x_{1}\right) & \cdots & y_{1} y_{n} \varphi\left(x_{1}\right) \varphi\left(x_{n}\right) \\
\vdots & \vdots & \ddots & \vdots \\
y_{n} & y_{n} y_{1} \varphi\left(x_{n}\right) \varphi\left(x_{1}\right) & \cdots & y_{n} y_{n} \varphi\left(x_{n}\right) \varphi\left(x_{n}\right)
\end{array}\right] \times\left[\begin{array}{c}
b \\
a_{1} \\
\vdots \\
a_{n}
\end{array}\right]=\left[\begin{array}{c}
0 \\
1 \\
\vdots \\
1
\end{array}\right] .
$$

Define the mapping function in equation (5), $\varphi\left(x_{i}\right)$ $\varphi\left(x_{j}\right)=K\left(x_{i}, x_{j}\right)$. Then, the function $K\left(x_{i}, x_{j}\right)$ can be defined as the kernel function of the LS-SVM model. After the simplification of the linear equations, the LSSVM model mathematical expression can be obtained, as shown in the following equation:

$$
f(x)=\sum_{i=1}^{n} a_{i} K\left(x_{i}, x\right)+b
$$

where $K\left(x_{i}, x\right)$ represents the kernel function of LS-SVM.

There are many kinds of kernel functions, including linear kernel function, polynomial kernel function, sigmoid kernel function, and Gaussian radial basis kernel function. Among them, the Gaussian radial basis kernel function is a kernel function that can realize nonlinear mapping. It has good performance for both large samples and small samples, with fewer kernel parameters and the most extensive application. Its mathematical representation is shown in the following equation:

$$
K\left(x_{i}, x\right)=\exp \left(\frac{-\left|x_{i}-x\right|^{2}}{\sigma^{2}}\right)
$$

where $\sigma$ represents the kernel parameter.

\section{Optimization of Support Vector Machine Model Parameters for MR Dampers}

According to the above theoretical model, the kernel parameter $\sigma$ in the support vector machine plays a key role in selecting the optimal hyperplane, while the penalty factor $c$ balances model complexity and training error. Therefore, the optimal values of these two parameters must be determined in the training process of the model [19] so that the established model of the MR damper can have high accuracy. As a global optimization algorithm, the genetic algorithm has also been applied to parameter optimization of support vector machines, and good results have been obtained $[20,21]$. The specific optimization process is shown in Figure 2.

During the optimization calculation, the selection interval of the penalty factor $c$ and kernel parameter $\sigma$ is $(0,100)$ and $(0,10)$, respectively. For the crossover calculation, the probability is 0.6 ; thus, the next generation of the population was formed. The mutation rate is arbitrary between 0.1 and 0.001 , and it was set at 0.03 in the paper to mutate individuals in the next generation of the population. The group size is set as 50 , and the maximum number of iterations is set as 500 . The fitness function is used as the evaluation index in the whole optimization process, and the final calculation result is that the error of the model after optimization reaches the minimum value. Therefore, when the fitness function reaches the maximum value, it can be used as the reference at the end of optimization. The fitness function set in this paper is shown in the following equation:

$$
F(c, \sigma)=\frac{1}{\sum_{i=1}^{n}\left[y_{i}-f\left(x_{i}\right)\right]^{2}+e},
$$




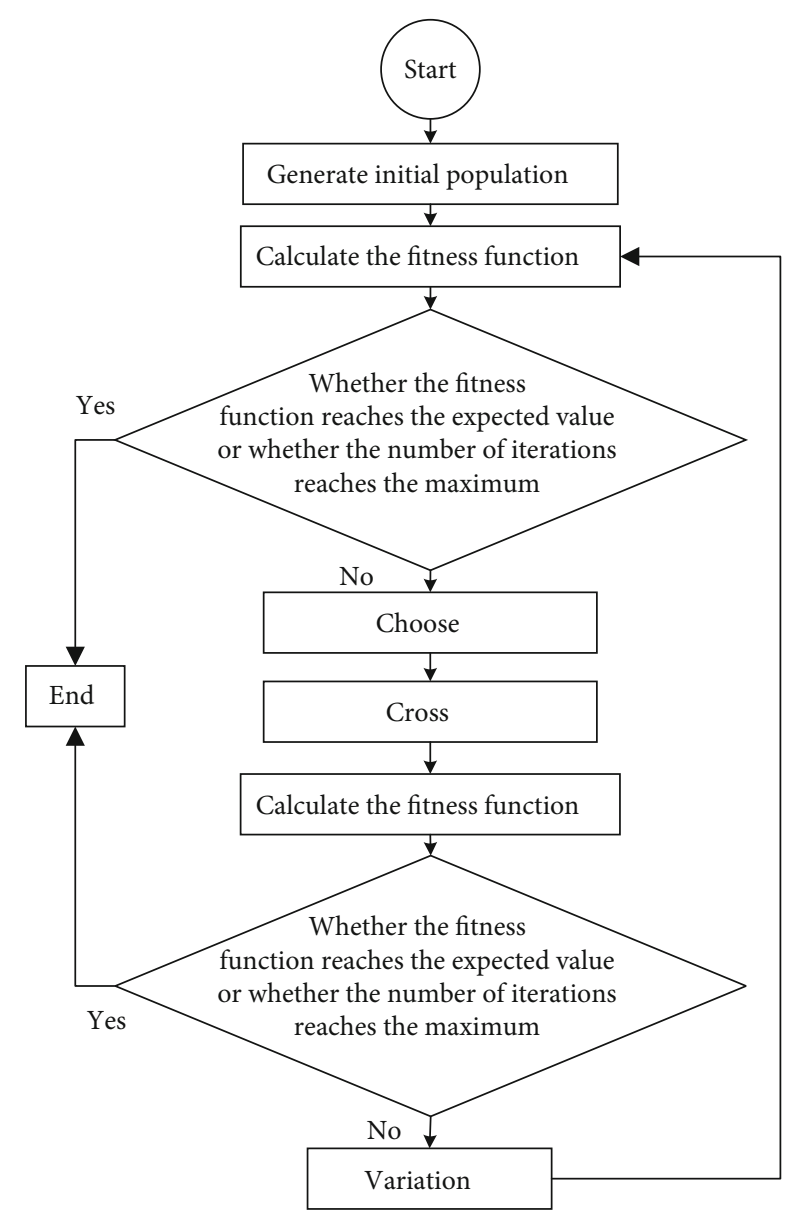

FIGURE 2: Schematic diagram of genetic algorithm optimization.

where $y_{i}$ represents the output obtained from the experiment and $f\left(x_{i}\right)$ represents the output of the LS-SVM training model. To avoid a zero denominator, set $e=10^{-6}$.

\section{Model Characteristic Analysis and Model Training Optimization of the MR Damper}

4.1. Acquisition and Analysis of Damping Characteristics of the MR Damper. The damping characteristic test system of the MR damper mainly includes the magnetorheological damper (type: BD-MRFD $\times 06 \times 4$; the maximum output force is $0.6 \mathrm{kN})$. The servo valve-controlled hydraulic control system is used as the loading channel of the magnetorheological damper, force sensor, displacement sensor, damper current controller, and computer. The basic working principle can be described as follows: set the current generator through the computer to the specific current value input of the magnetorheological damper and, at the same time, through the servo controller to control the servo valve in accordance with the specific loading amplitude and frequency of the load of the magnetorheological damper. In the process of loading, the damping force and corresponding displacement are measured by

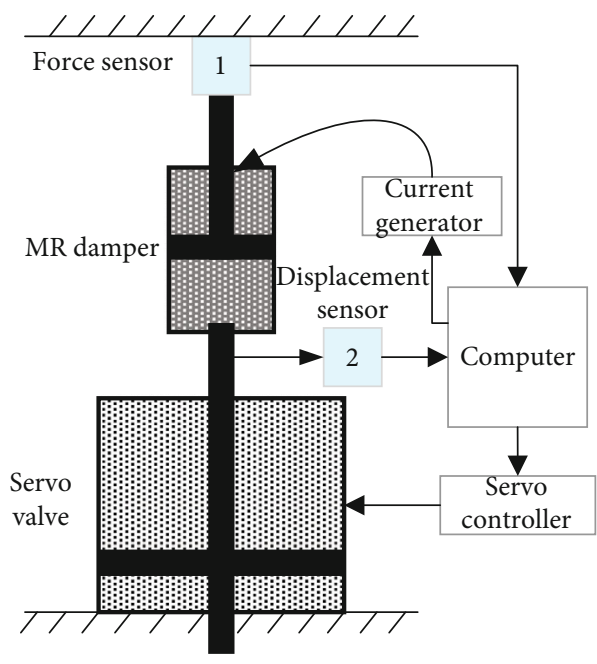

Figure 3: Schematic diagram of the damping characteristic test system for the MR damper.

the force sensor and displacement sensor. The test principle is shown in Figure 3.

In this paper, the characteristics of the damper are tested by using the sinusoidal signal as the excitation signal of the servo valve. The output amplitude of the servo valve is $15 \mathrm{~mm}$, and the frequency is $2 \mathrm{~Hz}$ and $4 \mathrm{~Hz}$, respectively. The control current of the magnetorheological damper is $0 \mathrm{~A}, 0.4 \mathrm{~A}$, and $0.8 \mathrm{~A}$, respectively. The curves of "damping force $F$-displacement $s$ " and "damping force $F$-velocity $v$ " of the MR damper are obtained through testing. Figures 4-6 show the comparison of the characteristics of the MR damper obtained under different conditions.

As can be seen from Figures 4-6, as the current value increases, the output damping force of the magnetorheological damper increases. With the increase of frequency, the damping force also increases to some extent. From the $F-v$ curves, it can be seen that there is an obvious hysteresis phenomenon in the low-speed region, and with the increase of frequency, the hysteresis phenomenon is intensified, which has a great influence on the accuracy of the model. In order to obtain an accurate control model, hysteresis nonlinearity must be effectively modeled.

4.2. Model Training and Parameter Optimization. It can be seen from the above characteristic analysis, damping force characteristics of the magnetorheological damper are affected by the current, frequency, amplitude. For the application of the least square support vector machine (LS-SVM) to establish the damping force model, the current and historical input displacements, velocity $v$, and current $I$ and the historical output under each characteristic curve are selected as the input of the SVM model, and the damping force of the current output is taken as the output of the model for model training, a total of 1260 training datasets. Meanwhile, the genetic algorithm is used to optimize the penalty factor $c$ and kernel parameter $\sigma$. In Figure 7, 50 sample reference 


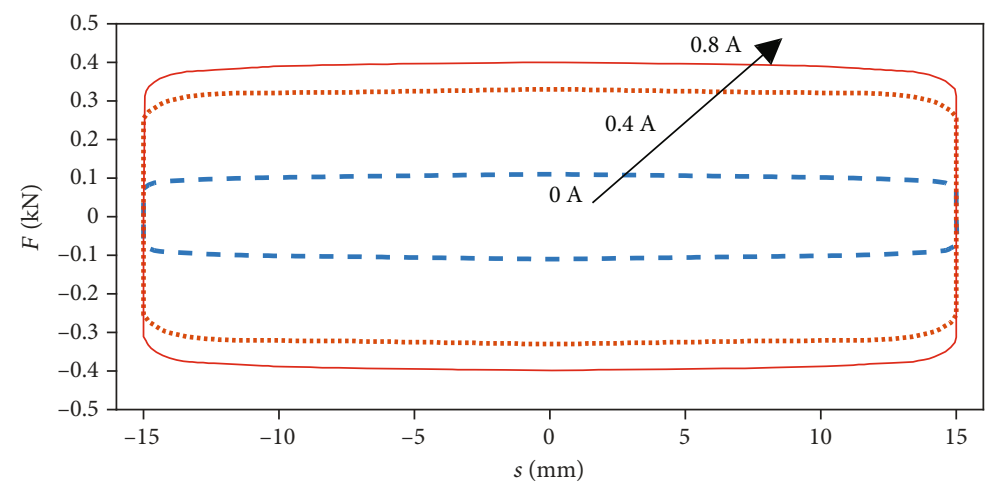

(a) F-s curves

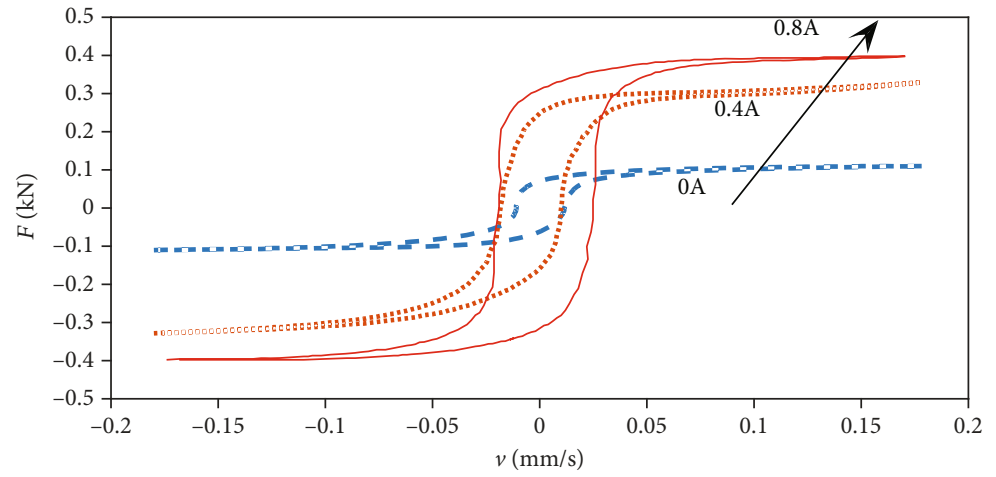

(b) $F$ - $v$ curves

Figure 4: $F-s$ curves and $F-v$ curves of different current values under $2 \mathrm{~Hz}$ and $15 \mathrm{~mm}$ excitation.

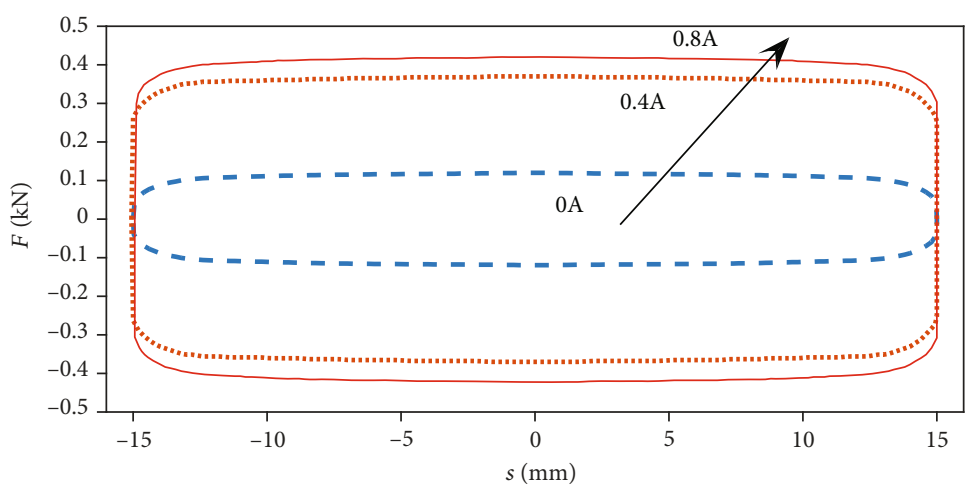

(a) F-s curves

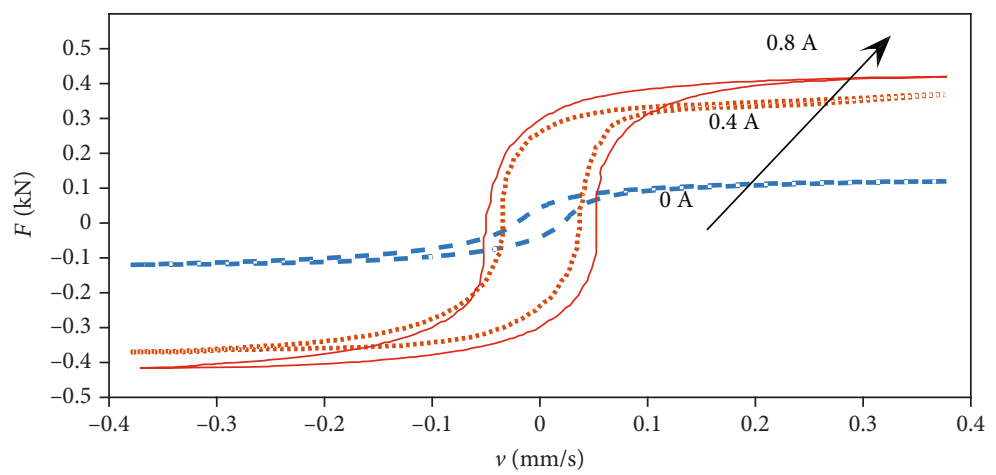

(b) $F-v$ curves

FIGURE 5: $F$-s curves and $F-v$ curves of different current values under $4 \mathrm{~Hz}$ and $15 \mathrm{~mm}$ excitation. 


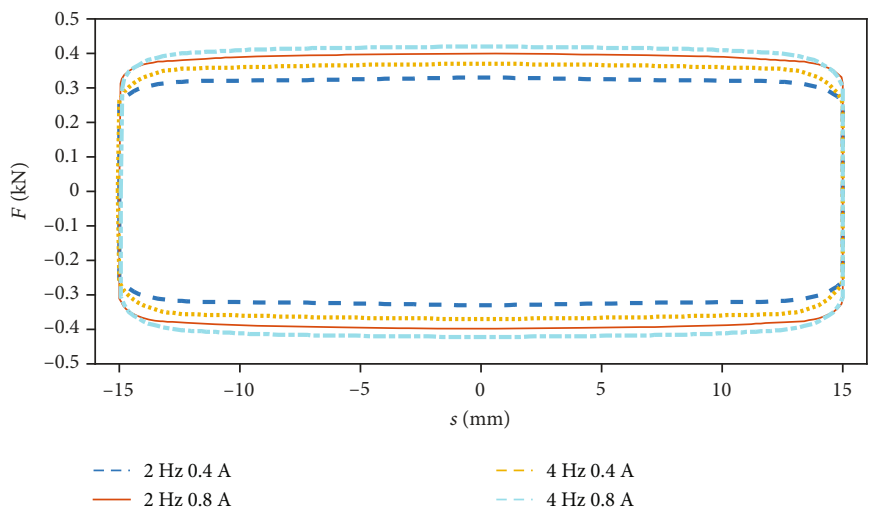

(a) F-s curves

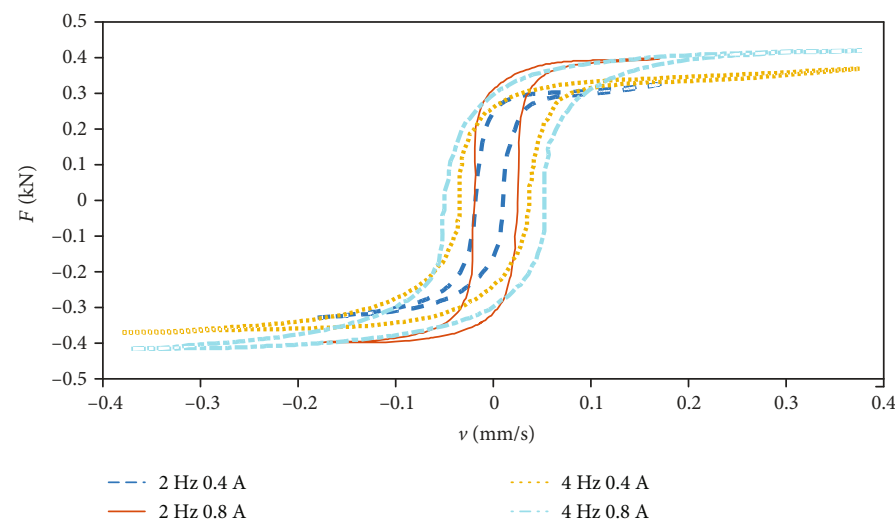

(b) $F-v$ curves

Figure 6: $F-s$ curves and $F-v$ curves at different input frequencies under $15 \mathrm{~mm}$ and $0.4 \mathrm{~A}$ and $0.8 \mathrm{~A}$.

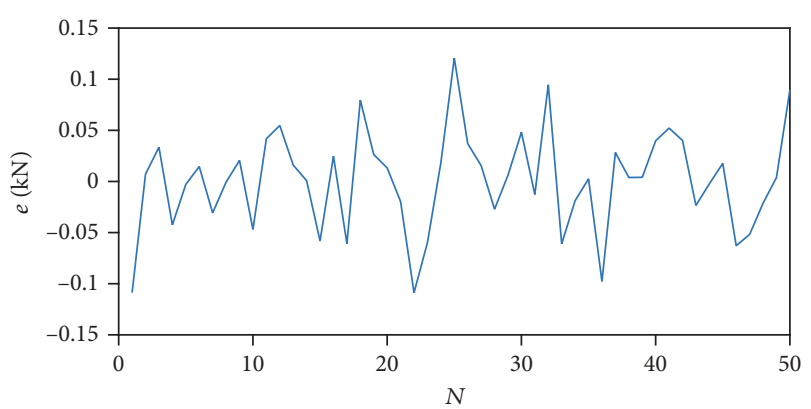

(a) Initial sample error

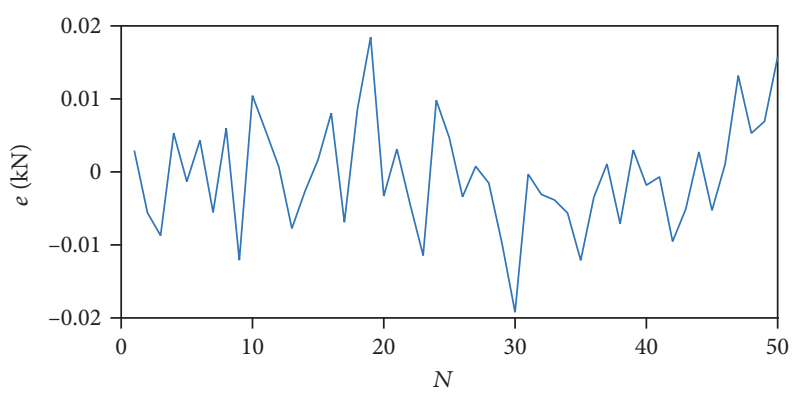

(b) Sample error after optimization

Figure 7: Changing trend of sample error in the optimization process.

points were randomly selected to observe the variation of the error of the genetic algorithm in the optimization process. In the figure, the $X$-coordinate $N$ is the number of sample points, and the $Y$-coordinate $e$ is the sample error. Figure 8 shows the convergence figure of the evaluation function in equation (8).

It can be seen from Figure 7 that under the initial penalty factor $c$ and the kernel parameter, the error of the reference sample point is large. After optimization, the error is significantly reduced. At this time, the maximum error in the sample point is around $0.02 \mathrm{kN}$, and most of them remain below

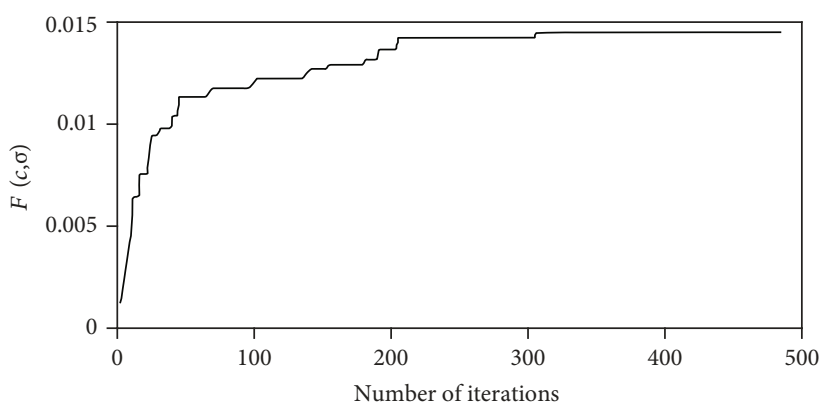

Figure 8: Convergence figure of the evaluation function. 


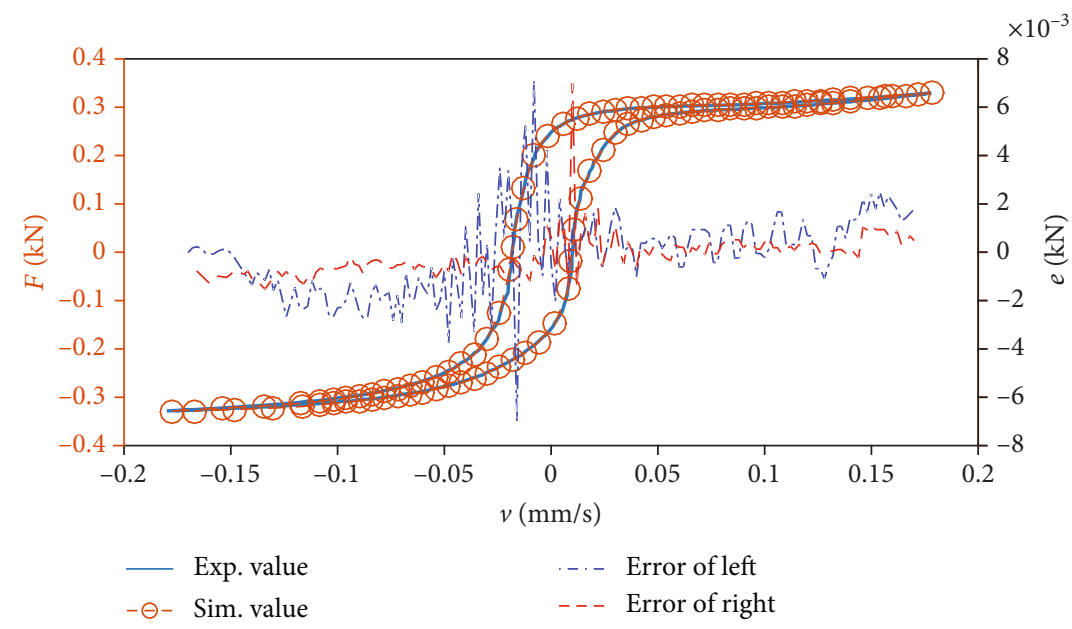

(a) $0.4 \mathrm{~A}$

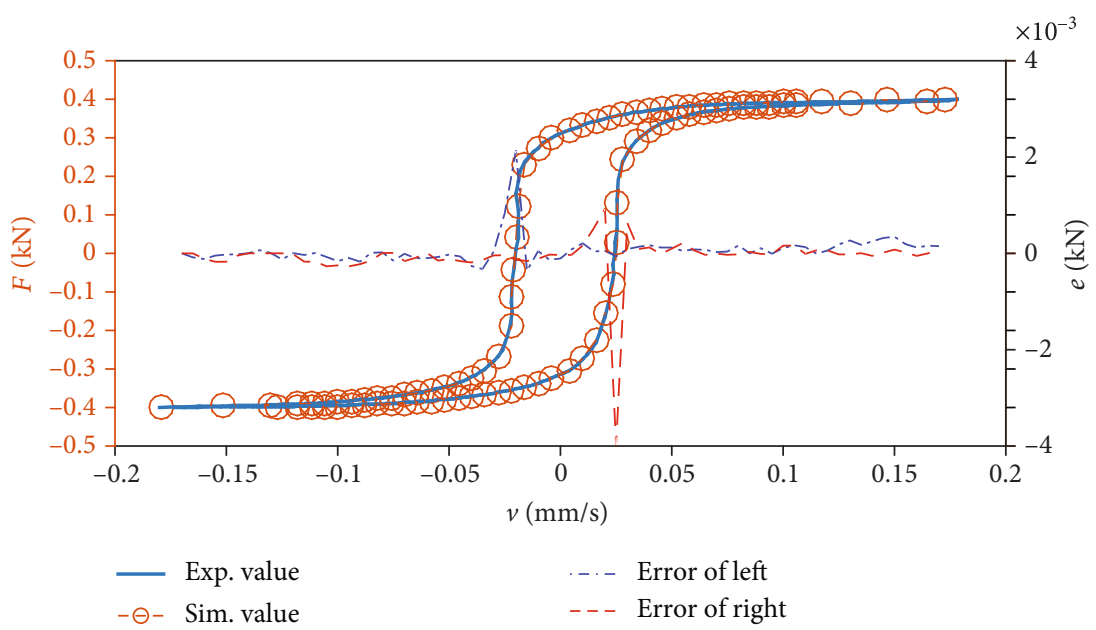

(b) $0.8 \mathrm{~A}$

Figure 9: $F-v$ comparison curves of different current values under excitation of $2 \mathrm{~Hz}$ and $15 \mathrm{~mm}$.

$0.01 \mathrm{kN}$, meeting the accuracy requirements of the model. After optimization, the penalty factor is $c=16.48$ and the kernel parameter is $\sigma=3.39$, and the least square support vector machine model of the magnetorheological damper is determined.

4.3. Model Validation. In order to verify the correctness of the above model, the above model is used for simulation analysis and comparison with experimental data. The input of the simulation model takes the current and historical input displacement $s$, velocity $v$, and current $I$ and the historical output as the input of the model to calculate the output damping force at the current moment. Figures 9 and 10 show the $F-v$ comparison diagram of partial simulation results and experimental data.

It can be seen from the comparison figure of $F-v$ under different current values and different frequencies of excitation in Figures 9 and 10 that the MR damper model based on the least square support vector machine model has good accuracy. Its maximum error mainly occurs in the low-speed region, and the maximum error is within $10 \mathrm{~N}$, with an average error of around $0.89 \mathrm{~N}$, and the maximum relative error is $5.29 \%$ (Figure 9(a)), $4.74 \%$ (Figure 9(b)), 4.32\% (Figure 10(a)), and 5.65\% (Figure 10(b)), respectively. By comparing the results obtained in Reference [9], the maximum error is within $0.06 \mathrm{kN}$, and most of the error values are below $0.01 \mathrm{kN}$. After comparison, it is found that the accuracy of the LS-SVM-based magnetorheological damper model established in this paper is similar to that obtained by using the modified particle swarm optimization method, and the maximum error and average error are improved to a certain extent, which is higher than that obtained by the traditional particle swarm optimization algorithm. Meanwhile, the Mean Squared Error (MSE) was calculated and compared with Reference [16], and the results are shown in Table 1.

As can be seen from the table, the results are similar to those in Reference [16] which verifies the correctness and effectiveness of the model presented in this paper. 


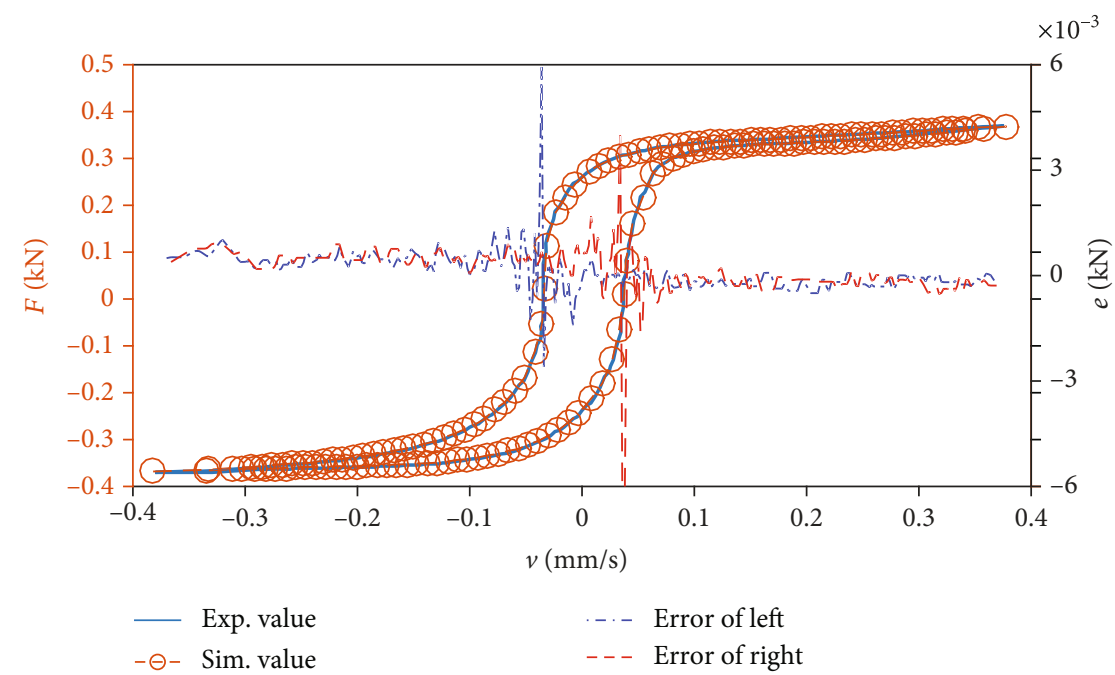

(a) $0.4 \mathrm{~A}$

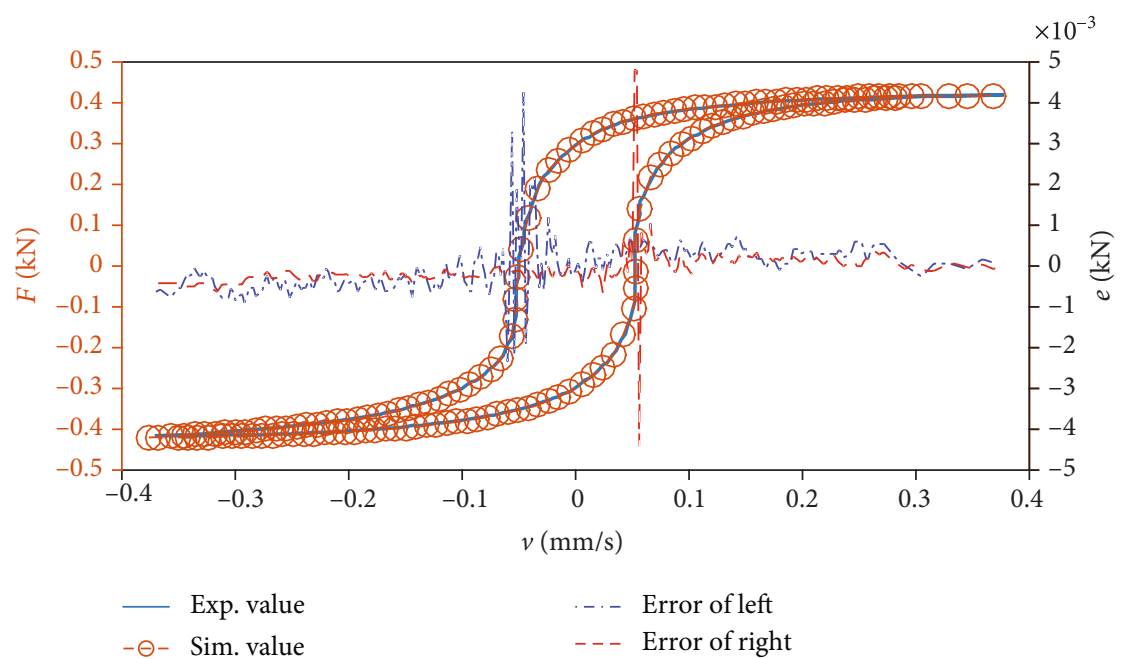

(b) $0.8 \mathrm{~A}$

Figure 10: F-v comparison curves of different current values under excitation of $4 \mathrm{~Hz}$ and $15 \mathrm{~mm}$.

TABLE 1: MSE results.

\begin{tabular}{lccc}
\hline & & $2 \mathrm{~Hz}$ & $4 \mathrm{~Hz}$ \\
\hline \multirow{2}{*}{ GA-LS-SVM } & $0.4 \mathrm{~A}$ & 23.5104 & 16.1274 \\
& $0.8 \mathrm{~A}$ & 17.6783 & 13.4586 \\
SVR-GA in Reference [16] & 19.3005 & 13.3626 \\
\hline
\end{tabular}

\section{Conclusions}

(1) The theoretical model of the magnetorheological damper is established based on the theory of the least square support vector machine, and the optimal value of the penalty factor $c$ and kernel parameter $\sigma$ is optimized by the genetic algorithm

(2) Through the experiment to obtain the characteristic curve of the magnetorheological damper, the current and historical input displacement $s$, velocity $v$, and current $I$ and the historical output of the characteristic curve were selected as the input of the least square support vector machine model, and the damping force of the current output was taken as the output for the model training. Meanwhile, the genetic algorithm was used for parameter optimization to obtain the optimal calculation model

(3) Through comparative analysis of the simulation value and experimental value, and comparison with other works of literature, it is found that the MR damper model based on the least square support vector machine has high accuracy and is an effective calculation model

(4) The following research mainly includes frequencydependent MR model construction and inverse model control algorithm research so as to be applied in the engineering field 


\section{Data Availability}

The processed data required to reproduce these findings cannot be shared at this time as the data also forms part of an ongoing study.

\section{Conflicts of Interest}

The authors declared no potential conflicts of interest with respect to the research, authorship, and/or publication of this article.

\section{Acknowledgments}

This work was supported in part by the National Natural Science Foundation of China (Grant number 51805209), the Natural Science Foundation of Zhejiang Province (Grant numbers LQ19A020002 and LQ17E050010), and the Jiaxing public welfare research project (Grant number 2019AD32032).

\section{References}

[1] O. El-Khoury, C. Kim, A. Shafieezadeh, J. E. Hur, and G. H. Heo, "Mitigation of the seismic response of multi-span bridges using MR dampers: experimental study of a new SMC-based controller," Journal of Vibration and Control, vol. 24, no. 1, pp. 83-99, 2018.

[2] D. X. Phu, N. Quoc Hung, and S. B. Choi, "A novel adaptive controller featuring inversely fuzzified values with application to vibration control of magneto-rheological seat suspension system," Journal of Vibration and Control, vol. 24, no. 21, pp. 107754631774047-107754631775018, 2017.

[3] Z. Feng, Y. Shi, G. Zhang, X. Liu, and Y. Duan, "Design of nonfragile damping controller for stay cable-magnetorheological damper," Transactions of the Chinese Society for Agricultural Machinery, vol. 51, no. 5, pp. 411-420, 2020.

[4] X. Kong, B. Li, L. Quan, B. Yi, and Y. Zhang, "Study on dynamic Bingham-polynomial model of a MRF damper," Journal of Mechanical Engineering, vol. 53, no. 14, pp. 179186, 2017.

[5] S. R. Hong, N. M. Wereley, Y. T. Choi, and S. B. Choi, "Analytical and experimental validation of a nondimensional Bingham model for mixed-mode magnetorheological dampers," Journal of Sound and Vibration, vol. 312, no. 3, pp. 399-417, 2008.

[6] N. Aguirre, F. Ikhouane, J. Rodellar, and R. Christenson, "Parametric identification of the Dahl model for large scale MR dampers," Structural Control and Health Monitoring, vol. 19, no. 3, pp. 332-347, 2012.

[7] H. Zhu, X. Rui, F. Yang, W. Zhu, and M. Wei, “An efficient parameters identification method of normalized Bouc-Wen model for MR damper," Journal of Sound and Vibration, vol. 448, pp. 146-158, 2019.

[8] M. S. Miah, E. N. Chatzi, V. K. Dertimanis, and F. Weber, "Nonlinear modeling of a rotational MR damper via an enhanced Bouc-Wen model," Smart Materials and Structures, vol. 24, no. 10, article 105020, 2015.

[9] X. Zhao, S. Wu, and H. Pan, "A hybrid model of magnetorheological dampers based on generalized hysteretic biviscous operators," Journal of Intelligent Material Systems and Structures, vol. 29, no. 14, pp. 2979-2985, 2018.
[10] H. Peng, J. Zhang, Y. Liu, J. Zhang, Z. Peng, and Y. Sun, "MR damper's modeling based on improved dual-sigmoid model," Journal of Vibration and Shock, vol. 38, no. 15, pp. 216-222, 2019.

[11] Y.-L. Zhao and X. Zhao-Dong, "A hysteretic model considering Stribeck effect for small-scale magnetorheological damper," Smart Materials and Structures, vol. 27, pp. 1-14, 2018.

[12] Y. Yu, Y. Li, and J. Li, "Parameter identification and sensitivity analysis of an improved LuGre friction model for magnetorheological elastomer base isolator," Meccanica, vol. 50, no. 11, pp. 2691-2707, 2015.

[13] X.-X. Bai, F.-L. Cai, and P. Chen, "Resistor-capacitor (RC) operator-based hysteresis model for magnetorheological (MR) dampers," Mechanical Systems and Signal Processing, vol. 117, pp. 157-169, 2019.

[14] Y. Yu, Y. Li, J. Li, X. Gu, and S. Royel, "Nonzracterization of the MRE isolator using binary-coded discrete CSO and ELM," International Journal of Structural Stability and Dynamics, vol. 18, no. 8, pp. 1840007-1840024, 2018.

[15] Y. Yu, Y. Li, and J. Li, "Parameter identification of a novel strain stiffening model for magnetorheological elastomer base isolator utilizing enhanced particle swarm optimization," Journal of Intelligent Material Systems and Structures, vol. 26, no. 18, pp. 2446-2462, 2014.

[16] Y. Yu, Y. Li, and J. Li, "Forecasting hysteresis behaviours of magnetorheological elastomer base isolator utilizing a hybrid model based on support vector regression and improved particle swarm optimization," Smart Materials and Structures, vol. 24, no. 3, article 035025, 2015.

[17] A.-A. Zamani, S. Tavakoli, S. Etedali, and J. Sadeghi, "Modeling of a magneto-rheological damper: an improved multistate-dependent parameter estimation approach," Journal of Intelligent Material Systems and Structures, vol. 30, no. 8, pp. 1178-1188, 2019.

[18] V. Vapnik, The Nature of Statistical Learning Theory, Springer, New York, 1995.

[19] Y. Yu, C. Zhang, X. Gu, and Y. Cui, "Expansion prediction of alkali aggregate reactivity-affected concrete structures using a hybrid soft computing method," Neural Computing \& Applications, vol. 31, no. 12, pp. 8641-8660, 2019.

[20] Y. Yao, X. Shen, Q. Qiu et al., "Predicting the biochemical methane potential of organic waste with near-infrared reflectance spectroscopy based on GA-SVM," Spectroscopy and Spectral Analysis, vol. 40, no. 6, pp. 1857-1861, 2020.

[21] S. Wang, N. Liu, Q. Cheng et al., "Classification and identification of polycyclic aromatic hydrocarbons by three-dimensional fluorescence spectroscopy combined with GA-SVM," Spectroscopy and Spectral Analysis, vol. 40, no. 4, pp. 1149-1155, 2020. 